\title{
A Scalable Wireless Sensor Network (WSN) Based Architecture for Fire Disaster Monitoring in the Developing World
}

\author{
Emmanuel Lule \\ Makerere University/Department of Computer Science, Kampala, Uganda \\ Email: lule.emmanuel@cis.mak.ac.ug \\ Tonny Eddie Bulega \\ Makerere University/Department of Networks, Kampala, Uganda \\ Email: tbulega@cis.mak.ac.ug
}

\begin{abstract}
All over the world, there is a tremendous increase in disaster occurrences such as landslide, toxic gas pollutions and wild fires. Least developed countries like Uganda have taken minimal efforts in management and containment of such disasters on behalf of the local populace. The dangers of fires are as a result of lack of proper information about the location, intensity and rate of spread of fire. However, the use of WSN may be one of the alternative ways of reducing risks associated with spreading fires resulting into destruction of lives and property worth millions of shillings. Our research looks at fire monitoring using sensors deployed in an event field to detect possible precursors of a fire occurrence using a simulated approach of OPNET Modeler (Ver. 14.0). Mobile nodes were deployed in proximity of (500X500) $\mathrm{m}^{2}$ flat space moving at a uniform speed of $10 \mathrm{~ms}^{-1}$ for 30 minutes. Our simulations are based on ftp high priority traffic to reflect real time information transfer for analysis. This paper evaluates and discusses how sensed information can be transmitted through a network with minimal delay and proposes a scalable WSN architectural design based on protocol perspective i.e. AODV and DSR. Two QoS parameters have been considered i.e. delay and throughput. Our results show that AODV scheme has got a minimum delay of $0.2 \mathrm{~ms}-1$ and a maximum throughput of $1.7 \mathrm{Mbps}$. Hence it's highly recommended for monitoring fires in large open area settings compared to DSR that is suitable for smaller areas. Results show that DSR exhibits higher delays due to nodal congestion and high control overheads. However, introducing the proposed heterogeneous routing mechanism i.e. (AODV+DSR) into the network significantly improves the performance of DSR.
\end{abstract}

Index Terms-Wireless Sensor Networks (WSN), Environmental Disasters, Sensor Network Architecture.

\section{INTRODUCTION}

Management and containment of fire outbreaks in Uganda has always been had to control, resulting to severe economic loss to both government and private sector businesses. Uganda Police Force (UPF) annual report statistics of 2009 to 2013 compiled by the firefighting department indicate an increased rampant growth of fire occurrences in Uganda [1][2][18]. The causes of fire in Uganda are mainly attributed to electrical short circuits, suspected arson and overheating, besides global warming. Previous studies conducted indicated that most fire outbreaks occur within the central district of Kampala metropolitan area constituting of $70 \%$, compared to other regions within Uganda.

Ministry of Relief Disaster Preparedness \& Refuge (MoRDPF) till today has not constituted any contingency plan regarding deployment of fire monitoring systems to assist in early detection, management and control of fire outbreaks in Uganda. However, with progressive developments in low power technology and miniaturization of computing and sensing devices, means that natural environment can be realistically monitored of the potential dangers. Embedded computing systems through environmental science can provide prediction models for detection and analysis of real time environmental disasters like fires, floods [3][4]. Research in WSN has attracted a lot of potential in recent years, with promising potential in rescue missions as one of the most important element of public safety [5]. Our major focus is modelling fire because our environment is made up buildings of high value and people. Hence we need to know the characteristics and behavior of fire to fight it effectively. The research focused on two routing schemes based on IEEE 802.15.4 standard networks with emphasis on AODV and DSR and two QoS parameter i.e. delay and throughput. The geographical area of study was a section of an open market in Uganda. However, the security of sensors is not considered in the research. This paper proposes a WSN based architecture to monitor fire disasters in developing world based on two heterogeneous routing mechanisms i.e. AODV+DSR that shall efficiency deliver critical information to central police stations with minimal delays. The succeeding sections of this paper are organized as follows; Section 2 highlights application areas where WSNs have been 
applied. Section 3 describes the methods used in carrying out the research study. Section 4 describes how the experiment has been setup to ensure successful simulation results obtained. Section 5 discussions of results obtained, RREQ analysis, summary of results and proposed architecture or network layout. Section 6 conclusion and future works and section 7 recommendations.

\section{A. Application of Sensor Networks}

i. Monitoring of Environment: Floods, fires and landslides.

ii. Military or Security: By detecting enemy intrusion in battle zones or no fly zones.

iii. Context aware computing systems: Using sirens for alerting authorities in case of an incidence for quick action.

iv. Intelligent tracking applications: Tracking patients and doctors inside hospitals, monitoring industrial chain management and control.

\section{B. Why Wireless Sensor Networks?}

To provide information about the;

i. Location of fire

ii. Extent of spread of fire.

iii. Temperature/smoke at various locations of the fire field.

\section{Motivation}

Uganda is faced with a challenge of frequent fire outbreaks, according to Uganda Police statistics of 2009 to 2013 [1][2][18]. On average the fire department arrives at approximately $69 \%$ onsite and at this stage, the fire may have spread extensively. This calls for emergency fire monitoring and detection systems. Statistically, over $70 \%$ of the fires occur in Kampala metropolitan area [1][2], especially open markets are highly prone to wild fires because there densely populated.

Currently there is no contingency plan in the deployment of fire monitoring systems in Uganda to assist in early fire detection [6]. The existing method for firefighting in Uganda relies on people calling fire brigades to respond to fire emergencies. However, this process may result into unnecessary delays in alerting the fire department causing huge fire disasters. Fire detection systems that have been earlier proposed like, fire sprinklers or smoke detectors are effective for a limited indoor area i.e. within a room or a factory. With the ever increasing fire outbreaks in schools, market places and recreational centers, there is need for acquiring sensors systems to provide an early detection warning of fire before it spreads out to destroy the entire neighborhood. Secondly, sensor networks can provide reliable information to support and enhance existing firefighting strategies with minimal delay.

\section{Problem Description}

With a need to provide a cost effective fire monitoring and detection system, WSNs were introduced as an alternative to the more expensive traditional satellite detection systems that have long scanning cycles and cannot provide timely detection [7]. These networks however, predominantly depend on routing protocols for their critical information delivery during an emergency event response. Despite the breakthrough of sensor technology, it has not been very successful in wide area monitoring of fire conditions to reliably detect and transmit critical information to $\mathrm{BS}$ for fire prediction models. Because, such networks are highly resource constrained having a low bandwidth, a limited transmission range, and frequent node failure [8], [9]. Also, taking into account that fire has the ability to spread in unpredictable behavior causing destruction to both lives and property in the nearby surrounding. Hence in such critical applications like fire, data should be delivered in a certain period of time from the moment its sensed or else, it will be useless. Time constrained applications therefore, should have limited detection delay as an important factor to be considered. Previous research proposes state of the art systems that are not scalable and only monitor a relatively smaller geographical area, using a different approach providing data with longer delays [10]. In this research, we study the behavior of two routing schemes i.e. AODV and DSR in relation to WSN fire monitoring with varying network sizes, and seek to find the minimal monitoring delay at which fire can be detected in a given area. AODV and DSR routing protocols are unique to fire, because of their scalability factor and quickly respond to an event on demand. QoS parameters to be considered shall be, i.e. throughput and delay [12], [11].

\section{E. Significance of the study}

i. To provide a platform aiming at allowing a continuous monitoring of environmental disasters such as floods, fires or toxic gas pollutions at low cost and minimum delay, while optimizing a high throughput efficiency and reliability.

ii. To ensure QoS in monitoring systems using the available limited bandwidth, to reliably transmit and deliver critical information to the intended destination.

iii. Analytical results can be used by relevant departments as a policy making basis by which the department shall make the decision of firefighting or fire prevention.

\section{F. Significant Contributions}

i. Architecture to support existing firefighting techniques with minimal delay, while optimizing coverage of the monitored area.

ii. Introducing eterogeneous outing, lowers the end to end delay for DSR and tremendously

Increases the throughput efficiency for DSR. 
Fire and Flood Detection Systems: WSNs are deployed in the forests to detect the origin of forest fires. Weather sensors are used in flood detection to detect, predict and hence prevent floods. Some nodes are deployed in the environment for monitoring biodiversity. The Forest Fire Surveillance System (FFSS) [13], [14] was developed to monitor fires in mountains and to have an early fire alarm in real time. The system senses environmental state such as temperature, humidity, smoke and determines forest the fire risk level by formula. Early detection of heat is possible and this allows an early alarm in real time when the forest fire occurs, alerting people to extinguish forest fires before it grows. This prevents the economic loss and environmental damage. Similarly, a typical application of WSN for flood detection and prevention is the ALERT system [15] deployed in the US, rainfalls, water levels and weather sensors are used in this system to detect, predict and hence prevent floods. The sensors supply critical information to the central database system in a predefined way.

Water Distribution Monitoring System: In [16], proposes a WSN based solution for monitoring the water distribution network for purpose of pollution detection and further addresses propagation concerns to overcome fast fading effect. By considering various technical issues like power consumption, radio propagation models and routing protocols and sensor needed for different applications, a suitable analytical communication model was proposed. The researchers focus on the physical layer of the sensor network i.e. radio propagation to determine the appropriate path loss model.

\section{Methodology}

Data was collected by way of simulation using Opnet Modeler (Ver. 14.0) and Microsoft visual studio 2005 to provide an integrated development platform for $\mathrm{C} / \mathrm{C}++$ discrete event simulations compiler [17]. A simulation experiment consisting of sensor nodes distributed in an area of $(500 \times 500) \mathrm{m}^{2}$ was used to collect data and register events. AODV, DSR and the heterogeneous AODV+DSR were evaluated in Opnet using two performance parameters i.e. delay and throughput. The scalability factor was considered with varying increase in network size, and then a comparative study was done to compare the three routing schemes to determine the optimal minimum delay of the examined routing schemes.

\section{A. Experimental Setup}

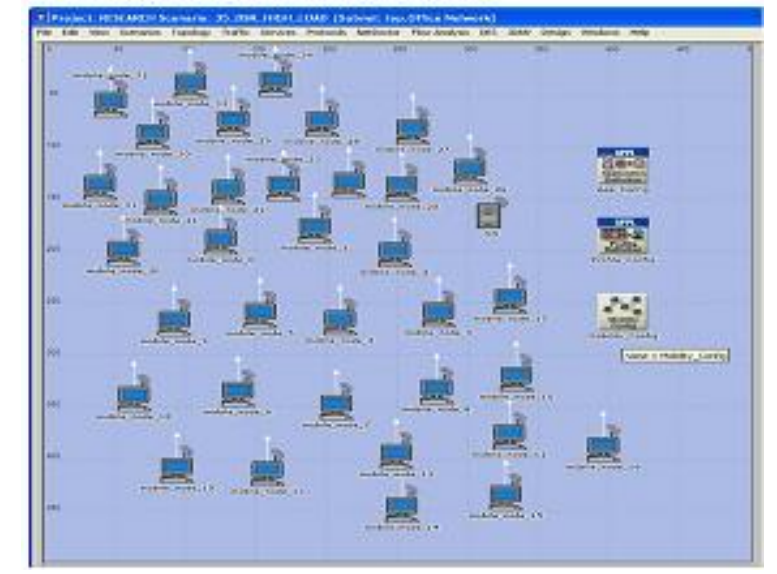

Fig 1: Setup of a Wireless Sensor Network Consisting of Mobile Nodes

The experiment was setup using a simulated approach of OPNET Modeler (Ver. 14.0). Mobile nodes were deployed in an area of $(500 \times 500) \mathrm{m}^{2}$ flat space to ensure effective communication between the nodes using a high data rate of $11 \mathrm{mbps}$. Our WLAN model was configured using application config object, i.e. ftp traffic source, profile config object, define the end-user profile for the ftp application, and then the mobility config object defining the free movement of nodes in the field with a uniform speed of $10 \mathrm{~m} / \mathrm{s}$. Each mobile node is configured using two routing schemes at different times i.e. AODV, DSR and the proposed heterogeneous AODV+DSR. We tested six different for each type of routing scheme, i.e 10 20, 35, 50, 80 and 100 nodes. Each of the deployed mobile node acts as a source, sending ftp traffic to the gateway within a cell (cluster). In our experiment all attribute values remained the same except the number of nodes $(\mathrm{N})$ varies. The routing protocols were changed to better understand the behaviour/response of the network with an increased node density. Each scenario is run for a duration equivalent to 30 minutes. Two QoS parameter were considered i.e, average throughput and end to end delay.

\section{B. Simulation Parameters}

Table 1: Simulation Parameters Considered.

\begin{tabular}{|l|l|}
\hline Parameter & Value \\
\hline Communication Protocols & AODV, DSR, \& AODV+DSR \\
\hline Simulation Time(sec) & 1800 approx. 30mins \\
\hline Simulation Area $\left(\mathrm{m}^{2}\right):$ & $(500 \times 500)$ \\
\hline Network Size & $10,20,35,50,80, \& 100$ nodes \\
\hline Type of Traffic & ftp traffic \\
\hline Speed & $0-10 \mathrm{~m} / \mathrm{s}$ \\
\hline Packet Size(bytes) & 512 \\
\hline Type of Nodes & Mobile Nodes \\
\hline QOS Parameters & Throughput, Delay \\
\hline Priority Traffic Load & High load \\
\hline Data Rate & 11 Mbps \\
\hline Transmit Power(Watts) & $0.005 W$ \\
\hline Mobility Model & Random Way Point \\
\hline
\end{tabular}




\section{DISCUSSION OF RESULTS}

\section{A. Using Average End to End Delay (s) Parameter}

Table 2: Delay (ms) results for each scenario having N- Number of Nodes

\begin{tabular}{|l|l||l|l||l|l||l|l|}
\hline Nodes & Protocol & $\begin{array}{l}\text { Min } \\
\text { Delay }\end{array}$ & $\mathbf{T}_{0}$ & $\begin{array}{l}\text { Max } \\
\text { Delay }\end{array}$ & $\mathrm{T}_{1}$ & $\begin{array}{l}\text { Avg. } \\
\text { Delay }\end{array}$ & $\begin{array}{l}\text { (\% } \\
\text { Delay) }\end{array}$ \\
\hline 10 & AODV & 0.572 & 1782 & 0.855 & 594 & 0.714 & 17.9 \\
\hline & DSR & 2.002 & 468 & 3.339 & 1782 & 2.671 & 66.8 \\
\hline & AODV+DSR & 0.272 & 468 & 0.425 & 1782 & 0.349 & 8.7 \\
\hline 20 & AODV & 0.376 & 1782 & 1.138 & 432 & 0.757 & 18.9 \\
\hline & DSR & 3.230 & 702 & 1.665 & 1332 & 2.448 & 61.2 \\
\hline & AODV+DSR & 0.239 & 432 & 0.286 & 1782 & 0.263 & 6.6 \\
\hline 35 & AODV & 0.299 & 1782 & 0.322 & 144 & 0.311 & 17.3 \\
\hline & DSR & 0.835 & 234 & 1.513 & 1386 & 1.174 & 65.2 \\
\hline & AODV+DSR & 0.315 & 594 & 0.763 & 720 & 0.593 & 29.9 \\
\hline 50 & AODV & 0.290 & 108 & 0.362 & 1584 & 2.326 & 8.2 \\
\hline & DSR & 0.815 & 1008 & 3.874 & 162 & 0.345 & 58.6 \\
\hline & AODV+DSR & 0.362 & 1584 & 0.814 & 386 & 0.588 & 14.7 \\
\hline 80 & AODV & 0.270 & 108 & 0.284 & 144 & 0.277 & 27.7 \\
\hline & DSR & 0.515 & 486 & 0.958 & 756 & 0.737 & 73.7 \\
\hline & AODV+DSR & 0.270 & 108 & 0.439 & 738 & 0.355 & 35.5 \\
\hline 100 & AODV & 0.263 & 144 & 0.282 & 1710 & 0.272 & 30.2 \\
\hline & DSR & 0.520 & 684 & 0.808 & 864 & 0.664 & 73.8 \\
\hline & AODV+DSR & 0.260 & 126 & 0.665 & 684 & 0.463 & 51.4 \\
\hline
\end{tabular}

Table 3: Throughput (Mbps) for each scenario having N-Number of Nodes

\begin{tabular}{|l|l||l|l||l|l||l|l|}
\hline $\mathbf{N}$ & Protocol & $\begin{array}{l}\text { Min } \\
\text { T-(kbps) }\end{array}$ & $\begin{array}{l}\mathbf{T}_{\mathbf{m}} \\
\text { in }\end{array}$ & $\begin{array}{l}\text { Max } \\
\text { T-(kbps) }\end{array}$ & $\begin{array}{l}\mathbf{T}_{\mathbf{m}} \\
\text { ax }\end{array}$ & $\begin{array}{l}\text { Avg. } \\
\text { T-(kbps) }\end{array}$ & $\begin{array}{l}\% \\
(\eta)\end{array}$ \\
\hline 10 & \multirow{2}{*}{ AODV } & 0 & 450 & 8.921 & 1638 & 4.460 & 49.6 \\
\hline & DSR & 0 & 450 & 6.064 & 1098 & 3.032 & 33.7 \\
\hline & AODV+DSR & 0 & 450 & 4.081 & 1116 & 2.041 & 22.7 \\
\hline 20 & AODV & 0 & 414 & 21.935 & 1602 & 10.968 & 45.7 \\
\hline & DSR & 0 & 342 & 3.962 & 432 & 1.981 & 8.3 \\
\hline & AODV+DSR & 0 & 432 & 12.998 & 810 & 6.499 & 27.1 \\
\hline 35 & AODV & 0 & 126 & 198.751 & 1566 & 99.376 & 49.7 \\
\hline & DSR & 0 & 198 & 9.336 & 1458 & 4.668 & 2.3 \\
\hline & AODV+DSR & 0 & 216 & 75.016 & 558 & 37.508 & 18.8 \\
\hline 50 & AODV & 0 & 90 & 532.536 & 1710 & 266.268 & 44.4 \\
\hline & DSR & 0 & 126 & 11.306 & 1638 & 5.653 & 0.9 \\
\hline & AODV+DSR & 0 & 378 & 163.085 & 1674 & 81.542 & 13.6 \\
\hline 80 & AODV & 0 & 90 & 1741.719 & 1206 & 870.860 & 48.4 \\
\hline & DSR & 0 & 90 & 25.380 & 918 & 12.690 & 0.7 \\
\hline & AODV+DSR & 0 & 90 & 714.035 & 288 & 357.018 & 19.8 \\
\hline 100 & AODV & 0 & 108 & 2790.712 & 1548 & 1395.356 & 46.5 \\
\hline & DSR & 0 & 108 & 28.174 & 1350 & 14.087 & 0.5 \\
\hline & AODV+DSR & 0 & 108 & 1139.217 & 504 & 569.608 & 19.0 \\
\hline
\end{tabular}

Time taken for the duration is $t=30$ minutes, $T_{0}$ and $T_{1}$ denotes the minimum and maximum delay occurrence observed at a given point respectively.

\section{B. Classification of Networks According to Size of Nodes;}
C. Average Delay for Selected Small, Large and Very Large Networks

Table 4: Summary of results for selected small, large and very large networks

\begin{tabular}{|l|l||l|l||l|}
\hline Protocol & Metric & $\begin{array}{l}\text { Small } \\
\text { Network }\end{array}$ & $\begin{array}{l}\text { Large } \\
\text { Network }\end{array}$ & $\begin{array}{l}\text { Very } \\
\text { Large } \\
\text { Network }\end{array}$ \\
\hline AODV & Min. Delay(ms) & 0376 & 0.274 & 0.271 \\
\hline & Throughput(bps) & $\begin{array}{l}21935, \\
\mathrm{~T}=1602 \mathrm{~s}\end{array}$ & $\begin{array}{l}532535, \\
\mathrm{~T}=1710 \mathrm{~s}\end{array}$ & $\begin{array}{l}2790711, \\
\mathrm{~T}=1548 \mathrm{~s}\end{array}$ \\
\hline DSR & Min. Delay(ms) & 0.323 & 0.815 & 0.520 \\
\hline & Throughput(bps) & $\begin{array}{l}3962, \\
\mathrm{~T}=432 \mathrm{~s}\end{array}$ & $\begin{array}{l}11436.15, \\
\mathrm{~T}=1602 \mathrm{~s}\end{array}$ & $\begin{array}{l}28103.84, \\
\mathrm{~T}=1170 \mathrm{~s}\end{array}$ \\
\hline AODV+DSR & Min. Delay(ms) & 0.239 & 0.354 & 0.260 \\
\hline & Throughput(bps) & $\begin{array}{l}12998.34, \\
\mathrm{~T}=810 \mathrm{~s}\end{array}$ & $\begin{array}{l}163084, \\
\mathrm{~T}=1674 \mathrm{~s}\end{array}$ & $\begin{array}{l}1139216.8, \\
\mathrm{~T}=504 \mathrm{~s}\end{array}$ \\
\hline
\end{tabular}

Where: $\mathrm{T}$ - Maximum Throughput (bps), time $\mathrm{T}$ (seconds), $\mathrm{N}$ - number of nodes deployed in the sensor field.

For $(\mathbf{N} \leq \mathbf{2 0})$, Small Network,

For $(\mathbf{N}>\mathbf{2 0})$ and $(\mathrm{N} \leq 50)$, Large Network and or ( $\mathbf{N} \geq \mathbf{1 0 0})$, Very Large Network.

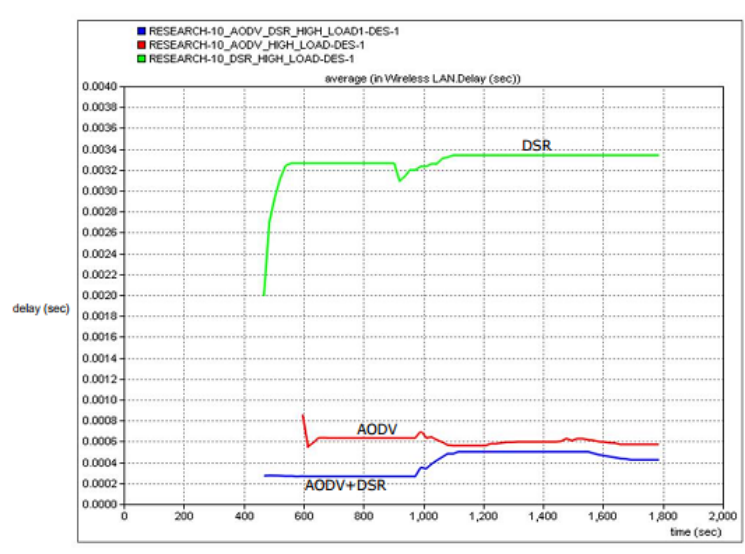

Fig 2: Effect of scalability on delay of AODV, DSR and the heterogeneous AODV+DSR for 10 mobiles nodes deployed in field.

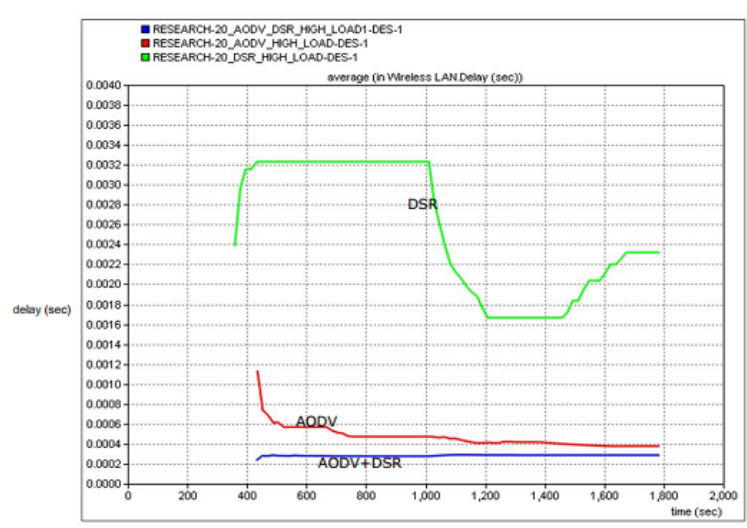

Fig 3: Effect of scalability on delay of AODV, DSR and the heterogeneous AODV+DSR for 20 mobiles nodes deployed in field.

In Fig. 2\&3, we observed a higher delay for DSR due to high network control overheads. However, we noted that the proposed heterogeneous AODV+DSR and standard AODV show minimum delays of approximately 0.27 and $0.38 \mathrm{~ms}$ because of a lower connection establishment and less overhead compared to standard DSR. 


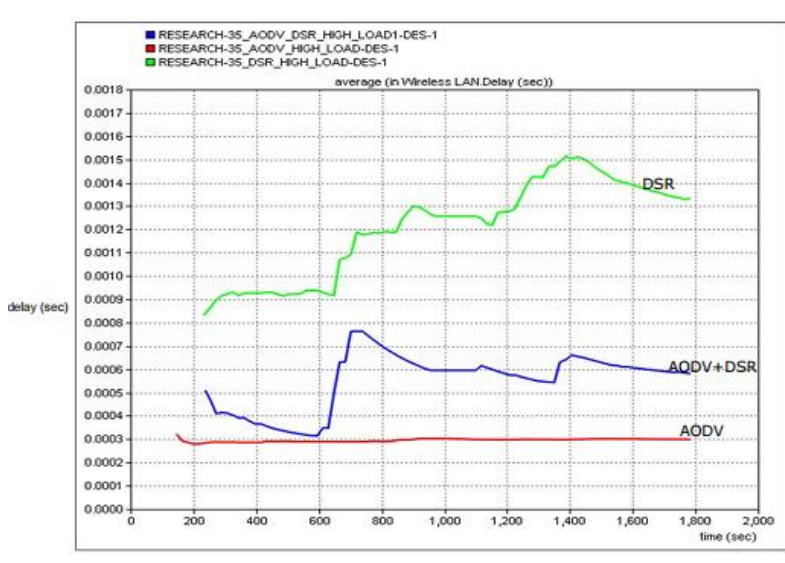

Fig 4: Effect of scalability on delay of AODV, DSR and the heterogeneous AODV+DSR for 35 mobiles nodes deployed in field.

In Fig. 4\&5, we increase the node density to test detection of a highly spreading wild fire. We further observe an increased in delay for DSR due to cached routes and unnecessary retransmissions. We also noted that the heterogeneous AODV+DSR have surpassed the delays in standard AODV for our settings. However, in Fig 4, we observe that a sudden delay reduction in DSR delay is due to the tendency that DSR uses multiple routes to minimize stale routes.

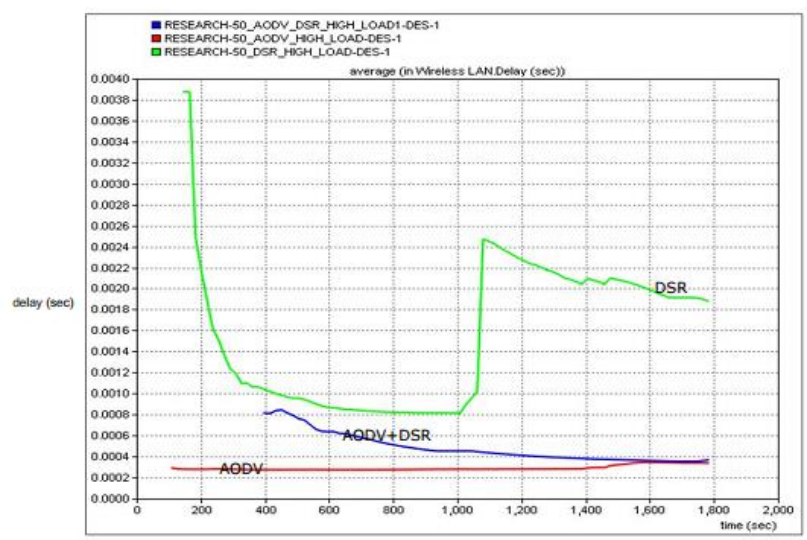

Fig 5: Effect of scalability on delay(s) of AODV, DSR and the heterogeneous AODV+DSR for 50 mobiles nodes deployed in field.

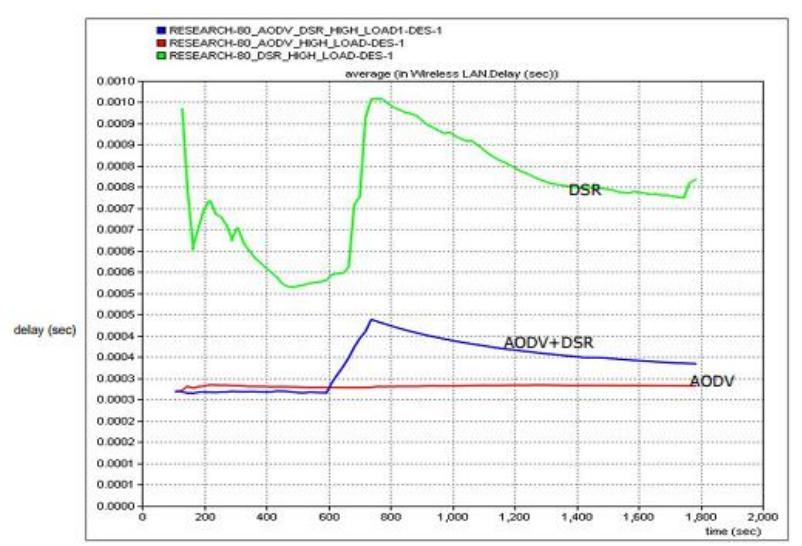

Fig 6: Effect of scalability on delay of AODV, DSR and the heterogeneous AODV+DSR for 80 mobiles nodes deployed in field

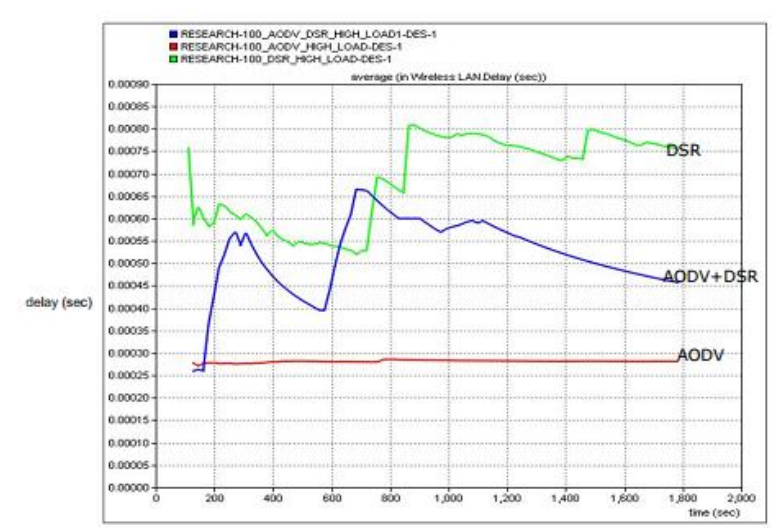

Fig 7: Effect of scalability on delay of AODV, DSR and the heterogeneous AODV+DSR for 100 mobiles nodes deployed in field.

In Fig. $6 \& 7$, we observed, higher delays are still observed in DSR with increased node density, as a result of excessive radio interference due to nodal congestion. Nevertheless, the overall performance of AODV yields lower delays with increased network size due to low traffic overheads and low connection delays hence high transmission success. The minimum tolerance limit of delay for effective monitoring was observed at approximately $0.2 \mathrm{~ms}$

\section{RREQ Allocation Analysis}

Processing and forwarding RREQ.

Pseudo Code for AODV RREQ Processing:

1. if(node listen equals RREQ packet) \{

2. if (same as forward in the near past) \{

3. discard; \}

4. else \{

5. entry for reverse path

6. update all();

7. set a valid flag for the route,

8. change life time (TTL) for the originator.

9. Update all routing table entries;

10. Increase (hop count +1) in RREQ packet; \}

11. If $(\mathrm{TTL}>1)\{$

12. decrease TTL field by one

13. If((Node is destination for RREQ) or (node has route to destination $)\{$

14. send RREP packet;

15. discard RREQ; \}

16. else \{

17. broadcast RREQ packet ; \} \} \}...

Pseudo Code for DSR RREQ Processing :

1. process RREQ packet;

2. if (route exists in table) \{

3. discard RREQ packet; \}

4. else \{

5. add RREQ to table;

6. if (destination node) \{

7. send RREP packet; $\}$

8. else \{

9. Rebroadcast RREQ packet ; $\}$..

Pseudo Code for heterogeneous AODV+DSR Processing:

1. if (node listen equals RREQ) \{

2. if (same as forward in the near past) \{

3. discard;

4. else \{

5. go to DSR: forward RREQ to intermediate DSR node

6. update:

7. update all () \{

8. sequence no;

9. set valid flag for the route

10. update all routing table entries 


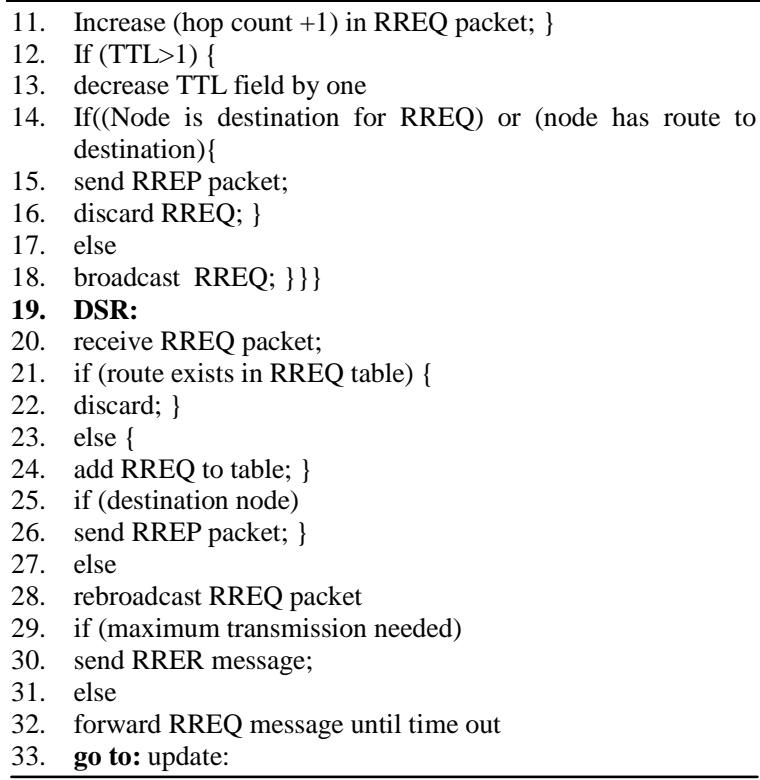

\section{E. Assumptions}

In proposing the heterogeneous $A O D V+D S R$ routing mechanism, the following assumptions were put into consideration such as;

i. Sensors of different routing mechanisms co-exist together in the same field to transmit critical traffic.

ii. In a heterogeneous environment, we assume that AODV sensor node initiates RREQ packets to an intermediate DSR node for path establishment.

iii. All mobile nodes move in a fixed field with a constant speed of $10 \mathrm{~m} / \mathrm{s}$.

\section{F. $\quad$ Using Throughput (bps) Parameter}

Throughput Efficiency $(\eta)=$ Average

Throughput/Total_Channel Capacity x 100\% (I)

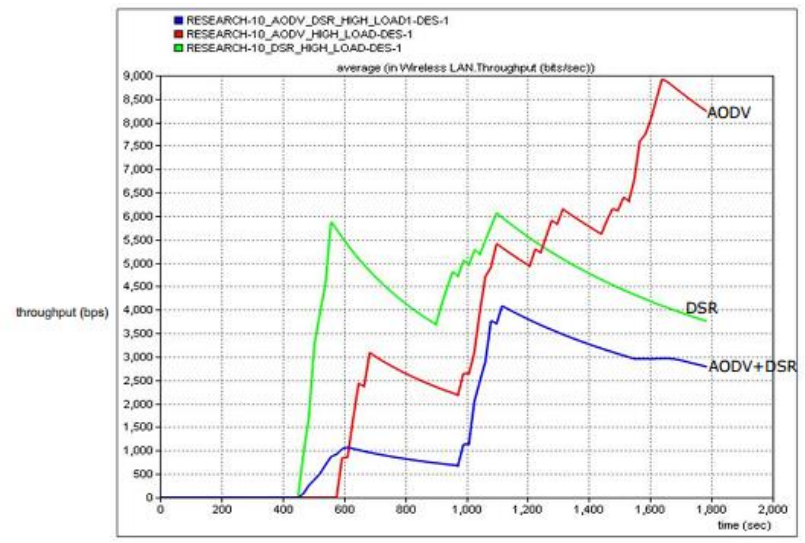

Fig 8: Effect of scalability on throughput (bps) of AODV, DSR and the heterogeneous AODV+DSR for 10 mobiles nodes deployed in field.

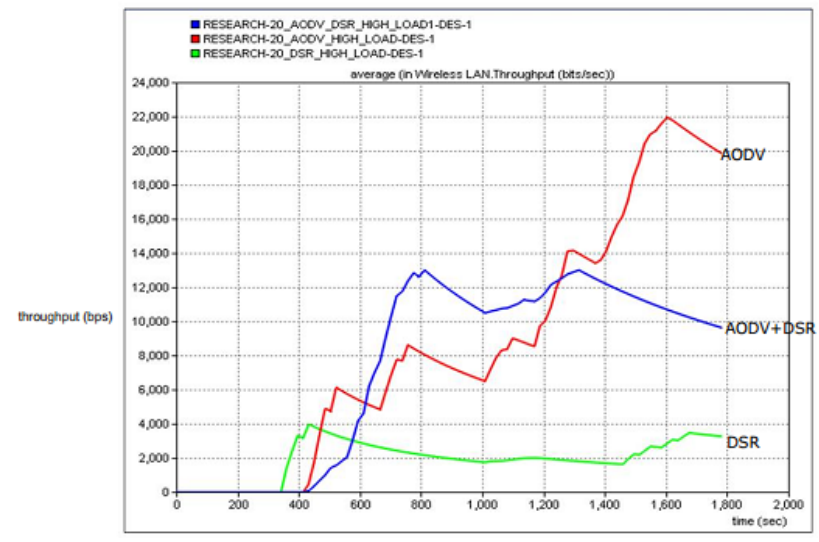

Fig 9: Effect of scalability on throughput (bps) of AODV, DSR and the heterogeneous AODV+DSR for 20 mobiles nodes deployed in field.

In Fig. 8 \& 9, we observed realized a higher throughput of $6 \mathrm{kbps}$ in a small network comprising of $(\mathrm{N}=10)$ nodes compared to AODV and the heterogeneous AODV+DSR. This is mainly because of less radio interference and less congestion from neighboring sources resulting into high throughput transmission efficiency. However, as the node density is increased we observed that the throughput of DSR is lowered due to high network control overheads as a result of congestion. While the throughput of both AODV and the heterogeneous AODV+DSR is significantly increased on the other hand.

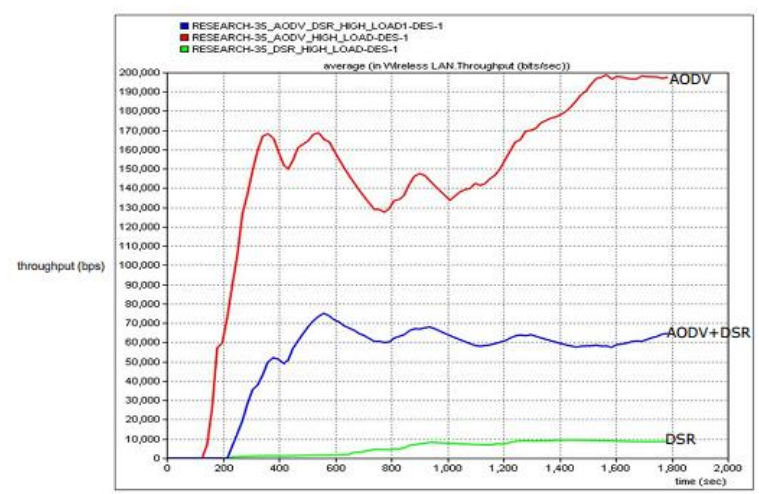

Fig 10: Effect of scalability on throughput (bps) of AODV, DSR and the heterogeneous AODV+DSR for 35 mobiles nodes deployed in field

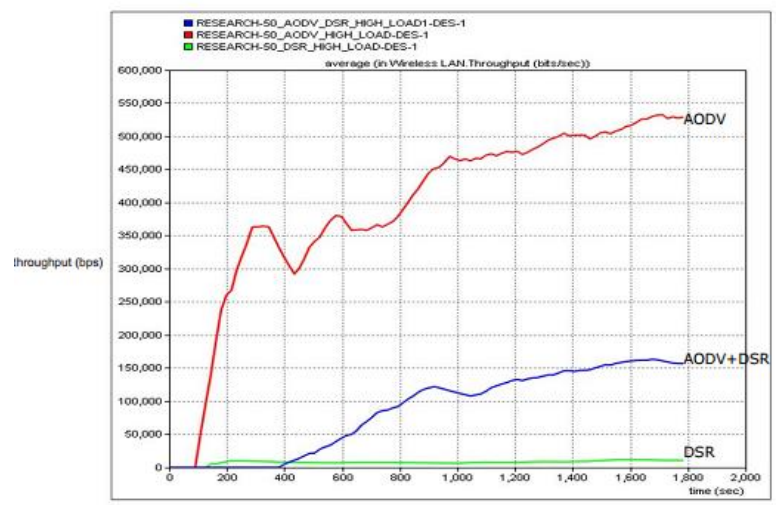

Fig 11: Effect of scalability on throughput (bps) of AODV, DSR and the heterogeneous AODV+DSR for 50 mobiles nodes deployed in field 
In Fig. 10 \& 11, we observed a further degradation in the throughput performance of DSR mainly due to nodal congestion and excessive radio interference from other node sources. On the other hand, AODV and the heterogeneous AODV+DSR yielded a high throughput performance than DSR due to low network overheads. In design, higher throughput translates into higher transmission success rate, hence lower delay. However, introducing heterogeneous routing into network significantly improves the throughput performance of DSR due to a reduction in control overheads.

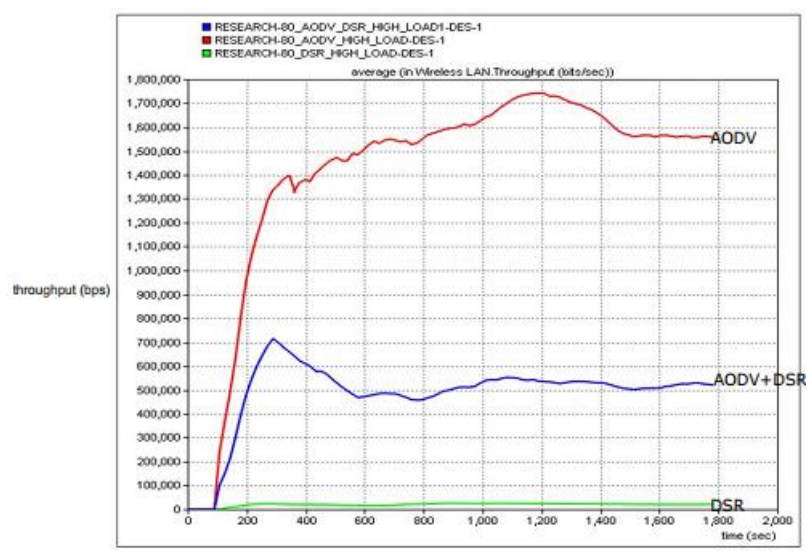

Fig 12: Effect of scalability on throughput (bps) of AODV, DSR and the heterogeneous AODV+DSR for 80 mobiles nodes deployed in field

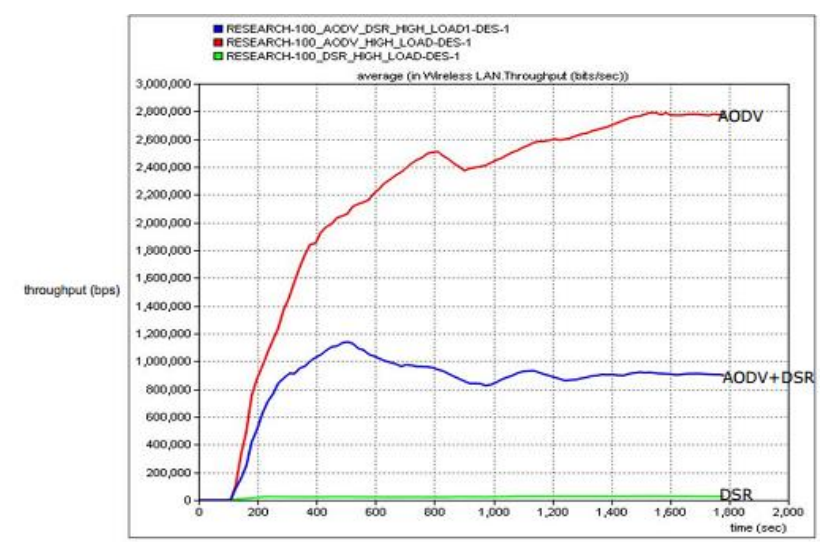

Fig 13: Effect of scalability on throughput (bps) of AODV, DSR and the heterogeneous AODV+DSR for 100 mobiles nodes deployed in field.

In Fig. 12 \& 13, we observed a further degradation in throughput performance for DSR due to excessive radio interference as a result of nodal congestion in the field. However, for AODV and heterogeneous AODV+DSR, we observe a progressive increase in network throughput performance compared to DSR. Increased node density means an increased number of bits transmitted per second. This however, results to a rapid degradation of DSR throughput until it reaches absolute zero as seen in Fig. 13.

From our findings it's observed that DSR is not scalable enough to monitor low and high intensity fires with extremely high and low spreading rate due to extended packet loss.

From our findings it's observed that; i. AODV provides a better throughput performance with minimal delays. This is mainly because AODV uses routing tables, one route per destination with destination sequence numbers to prevent loops and determine freshness of routes yielding a high transmission success than DSR. Hence nodes are assured of recent information which is only exchanged when needed. This implies the design of AODV can scale well to monitor extremely large areas without affecting its throughput efficiency as observed in Fig 8- 13, giving a throughput of up to $2.8 \mathrm{Mbps}$.

ii. It's also observed that increased network growth degrades the throughput performance of DSR. This implies that DSR is a poor routing protocol with lots of unnecessary delays due to caching. DSR has exhibited the least throughput performance and therefore not suitable for fire monitoring application. Also, higher delays in DSR are as a result of excessive interference due to congestion.

iii. Introducing heterogeneous routing (AODV+DSR) into the network, significantly increases the throughput performance of DSR to a relatively higher level of 1.1 Mbps ( $\mathrm{N}=100)$; hence improving performance due to low traffic overheads.

\section{G. Summary of Results}

Delay: Results show that AODV and heterogeneous AODV+DSR provide efficient information on fire occurrences with lower delays for small networks $(\mathrm{N} \leq 20)$ nodes. Increased node density does not affect the overall performance of AODV which is quite efficient for both high and low intensity fires; because it quickly adapts to changing environmental conditions. DSR is not suitable for monitoring fire conditions because it exhibits higher transmission delays resulting from potentially large control overheads. Hence, fire data collection requires a dense deployment of sensors with associated high data rates for effective data transfer between the source and destination with minimal delays. Hence a good protocol ought to be scalable enough and adaptive to topology changes in order to provide accurate information for fire prediction models. From results, AODV exhibited good scalability property behaviors compared to heterogeneous AODV+DSR and DSR schemes; hence it gives a lower delay performance for timely information transfer. In our network simulation experiment, we have observed that as the network density increases, the number of packets transmitted also increases as well; this brings about an increased delay in DSR due to high network overheads. This makes it inefficient for a fire monitoring application compared to AODV routing scheme. Therefore, the optimal network size to realize least delays were observed in a small network size of 10 to 25 nodes for a better communication traffic flow. In conclusion, our results show that AODV and heterogeneous AODV+DSR can provide efficient information on a fire occurrences with lower delays for small networks $(\mathrm{N} \leq 20)$ nodes. Increased node density does not affect the overall performance of AODV which is quite efficient for both 
high and low rate spreading fires; because it quickly adapts to changing environmental conditions. DSR is not suitable for monitoring fire conditions because it exhibits higher delays resulting from potentially large control overhead.

Throughput: Fire monitoring is a high data rate application; routing schemes should be designed to be adaptive to network topological changes. AODV yielded high throughput of $1.7 \mathrm{Mbps}$ due to less connection establishment. High throughput translates into high transmission rate and hence lower delay for AODV of approximately $0.2 \mathrm{~ms}$. A tradeoff between throughput utilization and detection delay is realized where lower delay in the network translates into higher throughput efficiency and vice versa. Early detection is crucial for a successful action to be taken by the firefighters; thanks to WSNs, fire brigades shall be able to recognize when a fire is started and be able to determine the rate of spread over the affected area.

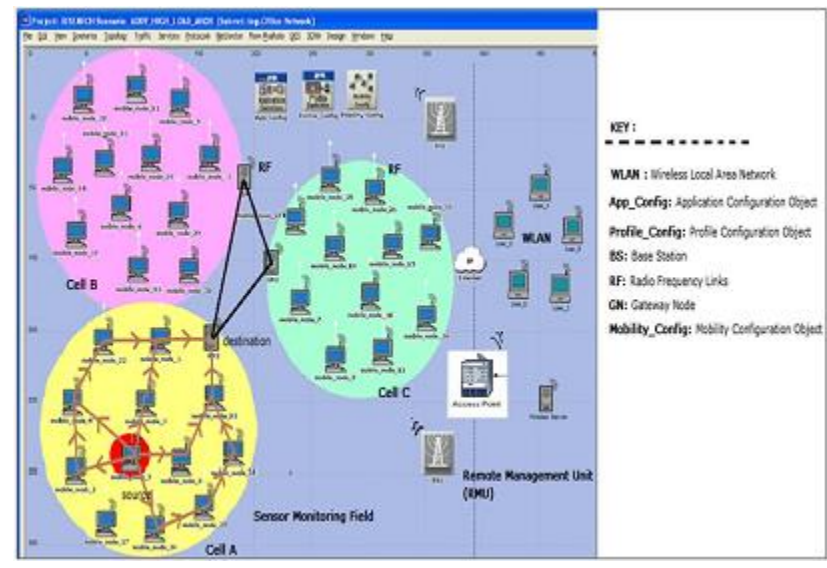

Fig. 14: Proposed WSN Architectural Field Design

The proposed WSN architectural design consists of randomly distributed mobile sensor nodes to monitor physical fire conditions within a targeted area. Nodes in the lower tier of the network are clustered to form cells in order to minimize energy consumption. Each cell (cluster) contains a fixed gateway sensor node (GN) that communicates state changes with the existing base station (BS) \& provides an interface between motes and host computers. The gateway node is fixed to provide a relatively stable location where other mobile sensors can calculate their location. Gateway nodes also have the capacity to aggregate network traffic from an array of sensor nodes within a cell, which traffic is further relayed to a sink. The BS and gateway connect through internet that acts as the bridge to the sensor field and the Remote Management Unit (RMU), sending traffic for modeling and analysis of the live situation of each fire field such that a possible action can be taken. The users of WLAN access the information through an Access Point (AP). The Application Config object specifies the standard application used i.e. ftp traffic, the Profile Config specifies the activity patterns of user(s) in terms of application used for a specific time and Mobility Config, defines the frequency of node movement within a specified area i.e. $10 \mathrm{~m} / \mathrm{s}$. When monitoring information is transmitted to the RMU directly, the network load becomes very heavy. Hence, in order to reduce the communication overhead and improve the stability of the network, a 2-tier hierarchical structure proposed is designed for our architecture. Sensor nodes were uniformly deployed in a field area of (500x500) $\mathrm{m}^{2}$, allowing them to communicate effectively within a defined communication range. In each cluster (or cell), a minimum number of relay nodes say, (where: $\mathbf{N}<\mathbf{2 0}$ ) can be deployed to avoid excess radio interference due to nodal congestion.

\section{H. Network Design Layout}

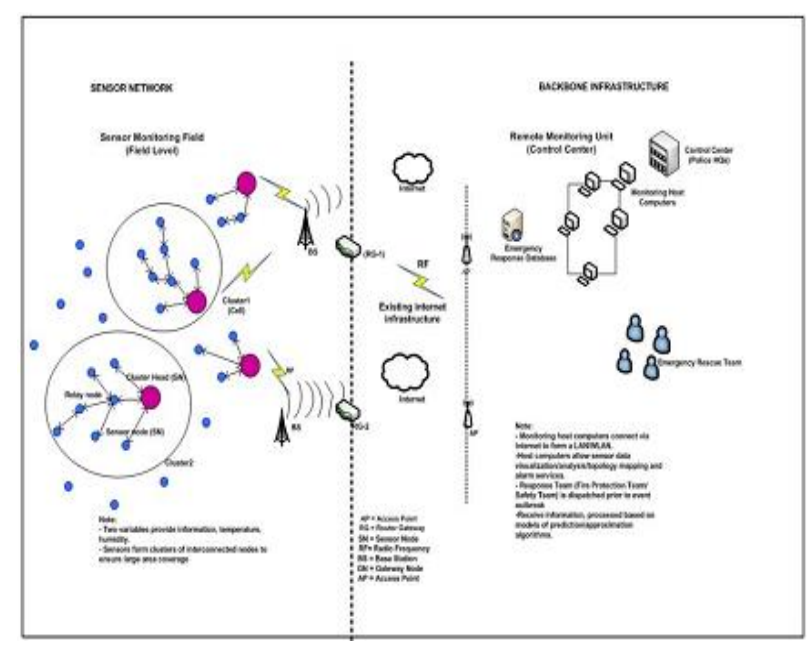

Fig. 15: Proposed Network Design Layout

\section{CONCLUSION AND FUtURE WORK}

In this paper, we proposed a scalable WSN based architecture for fire monitoring in a large open market that are highly prone to wild spreading fires in Uganda. The architecture is based on protocol design perspective to better understand how it can scale well in an area ranging from small to large settings to monitor critical events like fire. The architecture has been tested for scalability by increasing number of nodes to test for its performance effects on delay and throughput parameters. Our major contribution, was introducing a heterogeneous routing mechanism improved performance of DSR scheme for larger areas to be monitored effectively by lowering its delay and increasing its throughput efficiency.

Our focus in this research is centered towards mobile sensor nodes and the same protocols can be modeled and analyzed in a fixed scenario case study, if sensors are burnt due to fire or change due to climatic change, then it will be interesting to observe the behavior of the routing protocols in case of some failure of nodes and then check against the delay and throughput for all routing schemes. Our future works shall focus on studying the practical implementation of the proposed architectural design.

\section{RECOMMENDATIONS}


To government of Uganda:

i. Although the government of Uganda has tried to combat wild fires through the use of fire brigades, WSN intelligent systems which protect or detect against fire disasters have not been put in place as yet. Therefore, government should introduce fire warning systems to fight fires with minimum delays in order to reduce on the rates of life and property destructions. In this respect WSN would be ideal for such phenomena to enhance a controlled destruction of life and property for such disaster occurrences.

ii. Government through the Ministry of Relief Disaster Preparedness \& Refugee (MoRDPR) should sensitize its population on the most common causes and effects of wild fires as a public safety activity, and propose immediate safety precautions of avoiding such disasters in future.

iii. Government should train the police firefighting department in using WSN systems for disaster modeling and analysis such that quick response is taken prior to huge disastrous occurrences i.e. wild fires, stormy winds, toxic gas pollutions, landslides etc.

iv. Government together with the police fire department should make partnership with telecommunication companies to offer pre-disaster monitoring or detection services through mobile subscription or PDA to issue warning notices via sms as a public safety measure to alert local residents and dispatching firefighting crews.

\section{ACKNOWLEDGEMENT}

This work was done from Makerere University leading to an award of a MSc. in Data Communication \& Software Engineering, School of Computing \& Informatics Technology supported by the Belgium Development Corporation (BTC) scholarships program through Ministry of Education \& Sports, Republic of Uganda.

\section{REFERENCES}

[1] P. Musoke, "Police Report On fire outbreaks in Uganda", Available On www.upf.go.ug/fire_report_2011.

[2] P. Musoke, "Police Annual Emergency Statistics for all fire brigade systems" Uganda Police Available On www.upf.go.ug/fire_report_2010.

[3] Dr. M. Rakesh, "Wireless Sensor Networks for Disaster Monitoring: Amrita Center for Wireless Networks \& Applications" The $3^{\text {rd }}$ International Conference for Wireless Applications \& Research, 2009.

[4] W. Dargie, and C. Poellabatier Fundamentals of Wireless Networks, Theory and Practice", Wireless Communication \& Mobile Computing, 2009.

[5] K.Sha and W. Shi,'Using Wireless Sensor Applications for Fire Rescue Applications: Requirements \& Challenges", 2004.

[6] Disaster Preparedness and Refugees Ministry of Relief, Uganda national report and information on disaster risks reduction efforts," Work conference on disaster reduction in Kobe Hyogo Japan, 2005.

[7] Q. Shiguang Ju,"SMMS environmental monitoring and disaster management" available on www.apsco.int/smmsenvi:aspx, 2011.

[8] T. Lambrou and S. Felici-Castell, "Exploring Mobility in for Efficient Coverage in Sparse Wireless Sensor Networks," $10^{\text {th }}$ IEEE International Symposium in Wireless Personal Multimedia Communications, Jaipur India, 2007

[9] V. Rohit. "Applications of Wireless Sensor Networks for Environmental Monitoring and Development of an Energy Efficient Hierarchical Cluster Based Routing," Department of Electrical Engineering, National Institute of Technology, Rourkela), 2009.

[10] A. Casaca., "Wireless Sensor and Actuator Networks for Protection of Critical Infrastructures,'In the Proceedings of the 4th Monitoring Concertation Conference, 2010.

[11] D. Maltz, "The Dynamic Source Rouitng Protocol for Multi-Hop Ad-Hoc Networks," 1999.

[12] H. Heidemann Y.Xu and D. Estrin., "GeographyInformed Energy Conservation for Ad-hoc Routing," 7th Annual ACM/IEEE International Conference on Mobile Computing and Networks, 2001.

[13] Y. Her B. Son and J. Kim., "A Design and Implementation of Forest Fires Surveillance Monitoring System Based on Wireless Sensor Networks for South Korea Mountains," International Journal of Computer Science and Network Security, vol. 6, 2006.

[14] E. Yumis \& K. Ibrahim., "A Framework for Use of Wireless Sensor Networks in Forest Fire Detection\& Monitoring," In the Proceedings of the 4th International Conference on Computing and Wireless Networks Research, 2012.

[15] ALERT," www.alertsystems.org, 2011.

[16] Min Lin and Yan Wu, "Wireless Sensor Networks: Water Distribution Monitoring System," University of California, 2008.

[17] Cisco Systems, "Cisco Application Analysis Solution: A Standard Model User Guide," 2005.

[18] P. Musoke, "Annual Crime and Traffic/Road Safety Report", 2013 Available On www.upf.go.ug.

\section{APPENDIX.}

Table A1: Emergency incidences responded to by Uganda police fire brigade 2012 and 2013.

\begin{tabular}{|l|l|l|l|}
\hline S/No & Emergency & $\mathbf{2 0 1 3}$ & $\mathbf{2 0 1 2}$ \\
\hline $\mathbf{1 .}$ & Fire & 936 & 1,126 \\
\hline $\mathbf{2 .}$ & Rescue & 300 & 231 \\
\hline $\mathbf{3 .}$ & Fuel Tank Accidents & 44 & 15 \\
\hline $\mathbf{4 .}$ & Animal Rescue & 14 & 0 \\
\hline $\mathbf{5 .}$ & Others & 313 & 0 \\
\hline & Total & 1,607 & 1,372 \\
\hline
\end{tabular}

[Source: Annual Police Crime Report, 2013]

Table A2: Reports on victims of fire incidences in Uganda between 2012 and 2013

\begin{tabular}{|l|l|l|l|}
\hline S/No & Nature & $\mathbf{2 0 1 3}$ & $\mathbf{2 0 1 2}$ \\
\hline 1. & Injured & 56 & 28 \\
\hline 2. & Fatal & 62 & 22 \\
\hline & & 118 & 50 \\
\hline
\end{tabular}

[Source: Annual Police Crime Report, 2013] 
Table A3: Common premises affected by fire outbreaks in Uganda as per Uganda Police Reports of 2012 and 2013.

\begin{tabular}{|c|l|c|c|}
\hline \multirow{2}{*}{ S/No } & \multirow{2}{*}{ Premises } & \multicolumn{2}{|c|}{ Number } \\
\cline { 2 - 4 } & $\mathbf{2 0 1 2}$ & $\mathbf{2 0 1 3}$ \\
\hline $\mathbf{1 .}$ & Residential structures & 459 & 296 \\
\hline $\mathbf{2 .}$ & $\begin{array}{l}\text { Commercial (shops,kiosks } \\
\text { etc) }\end{array}$ & $\mathbf{2 6 3}$ & $\mathbf{1 4 0}$ \\
\hline $\mathbf{3 .}$ & $\begin{array}{l}\text { Educational institutions } \\
\text { (Schools \& Hostels) }\end{array}$ & 26 & 24 \\
\hline $\mathbf{4 .}$ & $\begin{array}{l}\text { Factories, workshop and } \\
\text { warehouses and stores. }\end{array}$ & 25 & 54 \\
\hline $\mathbf{5 .}$ & Automaobiles/garages & 13 & 52 \\
\hline $\mathbf{6 .}$ & $\begin{array}{l}\text { Wooden and grass thatched } \\
\text { Structures. }\end{array}$ & $\mathbf{1 0 6}$ & $\mathbf{9 7}$ \\
\hline $\mathbf{7 .}$ & $\begin{array}{l}\text { Farms, } \\
\text { hedges,plantations/forests,open } \\
\text { grounds/bushes. }\end{array}$ & 42 & 46 \\
\hline $\mathbf{8 .}$ & Markets & $\mathbf{0 7}$ & $\mathbf{2 0}$ \\
\hline $\mathbf{9 .}$ & Rubbish heaps and skips & 19 & 05 \\
\hline $\mathbf{1 0 .}$ & Electrical installations & 81 & 89 \\
\hline $\mathbf{1 1 .}$ & Fuel stations and tankers & 08 & 1 \\
\hline $\mathbf{1 2 .}$ & Hospitals & & $\mathbf{4}$ \\
\hline $\mathbf{1 3 .}$ & Office Premises & & 6 \\
\hline $\mathbf{1 4 .}$ & Recreation/Leisure Centers & 100 & $\mathbf{3 1}$ \\
\hline $\mathbf{1 5 .}$ & Others & $\mathbf{1 , 1 4 9}$ & $\mathbf{8 7 2}$ \\
\hline & Total & & \\
\hline $\mathbf{S}$ & Ans & & \\
\hline
\end{tabular}

[Source: Annual Police Crime Report, 2013]

\section{Authors' Profile}

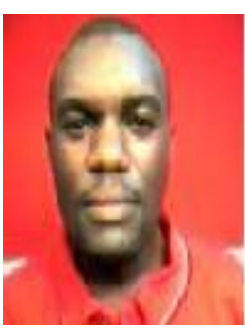

Emmanuel Lule: Is an Assistant Lecturer in the Department of Computer Science, Makerere University, Uganda $\mathrm{He}$ attained his BSc. in Information Technology \& Computing (BITC), Kyambogo University and a MSc. in Data Communication \& Software Engineering (MDCSE), Makerere University. Research interests, Wireless Networks and Computer Security.

Tonny Eddie Bulega: Is a Lecturer in the Department of Networks Makerere University. With BSc Eng from Makerere University and MEng/MIS and PHD in Engineering at South China University of Technology. Research interests include Wireless Sensor Networks and Capacity Planning.

Manuscript received June 23rd, 2014; revised July 05th, 2014; accepted August 18th, 2014.

How to cite this paper: Emmanuel Lule, Tonny Eddie Bulega,"A Scalable Wireless Sensor Network (WSN) Based Architecture for Fire Disaster Monitoring in the Developing World", IJCNIS, vol.7, no.2, pp.40-49, 2015.DOI: 10.5815/ijcnis.2015.02.05 